\title{
Rooting Out Pain
}

By Jack M. Gorman, MD

Pain is not a favorite topic of many physicians. Not too long ago, the Joint Commission for the Accreditation of Healthcare Organizations (JCAHO), the group that accredits most hospitals in the United States, made the evaluation and treatment of pain for every patient admitted to a hospital a key standard of its assessments. JCAHO found it necessary to explicitly demand that doctors ask patients if they are in pain and, if so, to do something about it.

It is unlikely that JCAHO has to remind doctors to check the blood pressure, temperature, or heart rate of newly admitted patients. These factors, of course, can be objectively measured and trigger a series of decision algorithms for which all physicians are well-trained. Pain, on the other hand, is largely subjective and often difficult to pin down. Indeed, like the emotional complaints that are the responsibility of psychiatrists or the complaints of dizziness that belong to neurologists, pain is often of neurogenic origin and therefore involves that most mysterious of all organs, the brain. Without firm guidelines for response, physicians are notorious for under-treating pain. Saying pain is "all in the head" is not exactly incorrect, but it is not a great excuse for dismissing the severity of a patient's suffering and despite studies to the contrary, doctors often still believe that postsurgical patients will become easily addicted to opiate analgesics such as morphine.

Given these concerns, the articles in this month's CNS Spectrums are welcome because they give us insight into what is known about the biology and therapy of a particularly vexing type of pain, neuropathic pain. Dan Buskila, MD, and colleagues tackle one form of neuopathic pain, the common but perplexing condition known as fibromyalgia. They review evidence that fibromyalgia and related conditions, such as chronic fatigue syndrome and irritable bowel syndrome, may share common underlying genetic factors. These factors particularly appear to involve neurotransmitter systems, such as serotonin and dopamine, commonly implicated in psychiatric and neurological disorders.

Martin Offenbaecher, MD, MPH, and Manfred Ackenheil, MD, review some of the available or nearly available treatments for neuropathic pain and fibromyalgia, some of which involve the dopamine and serotonin neurotransmitter systems, including antidepressants and possibly anticonvulsants.
Surprisingly, they assert that opiate analgesics are not particularly effective for neuropathic pain.

Moving to more experimental treatments, Walter Zieglgänsberger, $\mathrm{MD}$, and colleagues note that chronic pain involves a variety of biological processes including changes in the excitability of nociceptors, inflammation, and novel gene expression. These processes then become targets for a host of new molecular strategies aimed at treating neuropathic pain at its source.

A bit off the beaten track but ultimately relevant to neuropathic pain is the theoretical paper by $\mathrm{H}$. Stefan Bracha, MD, and colleagues. They speculate that the commonly observed clinical problem of teeth clenching, which is said by these authors to be related to several chronic pain conditions, including fibromyalgia, represents an evolutionarily conserved behavior. During the development of Homo sapiens, they assert, jaw clenching may have adaptively strengthened masseter and temporalis muscles and sharpened teeth necessary for biting during battle and for tearing apart raw meat during meals. How the alleles that the authors believe are associated with jaw clenching in $8 \%$ of the modern human population wound up being maintained is a topic for another set of speculations.

We also have a review of the treatment of psychotic depression by Stephen Stahl, MD, PhD. He notes several papers that have found fluvoxamine uniquely able to treat psychotic depression as monotherapy. Most other antidepressants must be combined with an antipsychotic to work but because fluvoxamine has particular affinity for the $\sigma$ receptor, Dr. Stahlwonders if this may underlie its supposed unique efficacy for psychotic depression. Notwithstanding the debate about whether fluvoxamine really is useful as monotherapy for psychotic depression, Dr. Stahl's idea is important as it provides a novel target for the treatment of psychotic mood disorders.

We hope that this issue of CNS Spectrums will fortify physicians when they inevitably confront patients complaining of chronic pain. There are many available treatments and, based on some exciting science, many more on the way. Soon, it will no longer be necessary for an accrediting agency to remind us to treat pain. CNS 

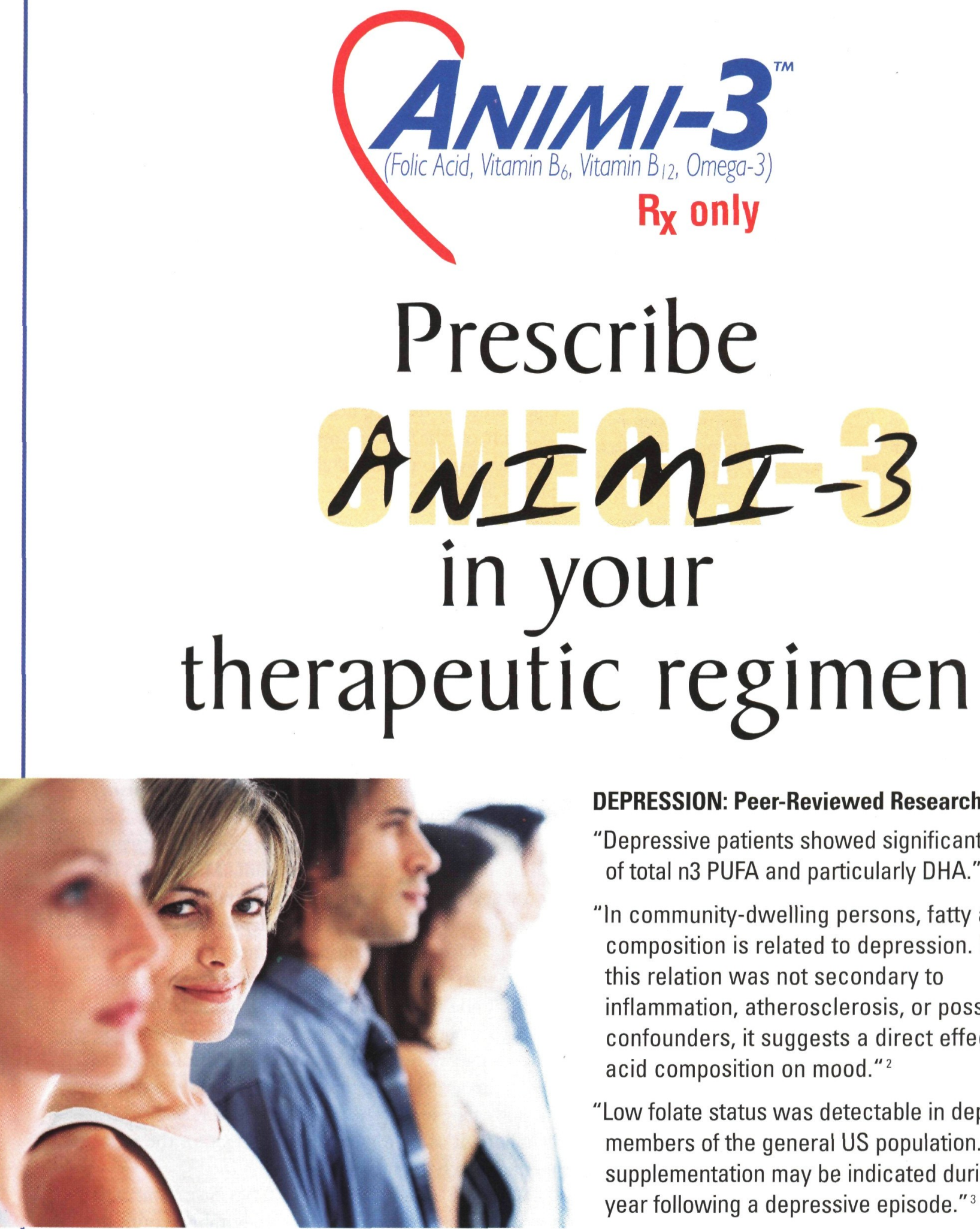

DEPRESSION: Peer-Reviewed Research Findings

“Depressive patients showed significant depletions of total n3 PUFA and particularly DHA."

"In community-dwelling persons, fatty acid composition is related to depression. Because this relation was not secondary to inflammation, atherosclerosis, or possible confounders, it suggests a direct effect of fatty acid composition on mood." ${ }^{2}$

“Low folate status was detectable in depressed members of the general US population. Folate supplementation may be indicated during the year following a depressive episode." ${ }^{3}$

Consider ANIMI-3 with none of the potentially significant adverse events of other agents.

Please see prescribing information on last page of this ad.

1 Peet, M., et al. Depletion of Omega-3 Fatty Acid Levels in Red Blood Cell Membranes of Depressive Patients. Biol Psychiatry 1998:43:315-19.

2 Tiemeier, H. et al. Plasma fatty acid composition and depression are associated in the elderly: the Rotterdam Study. Am J Clin Nutr 2003;78:40-6.

3 Morris, M.S., et al. Depression and Folate Status in the US Population. Psychother and Psychosom 2003;72:80-7. 


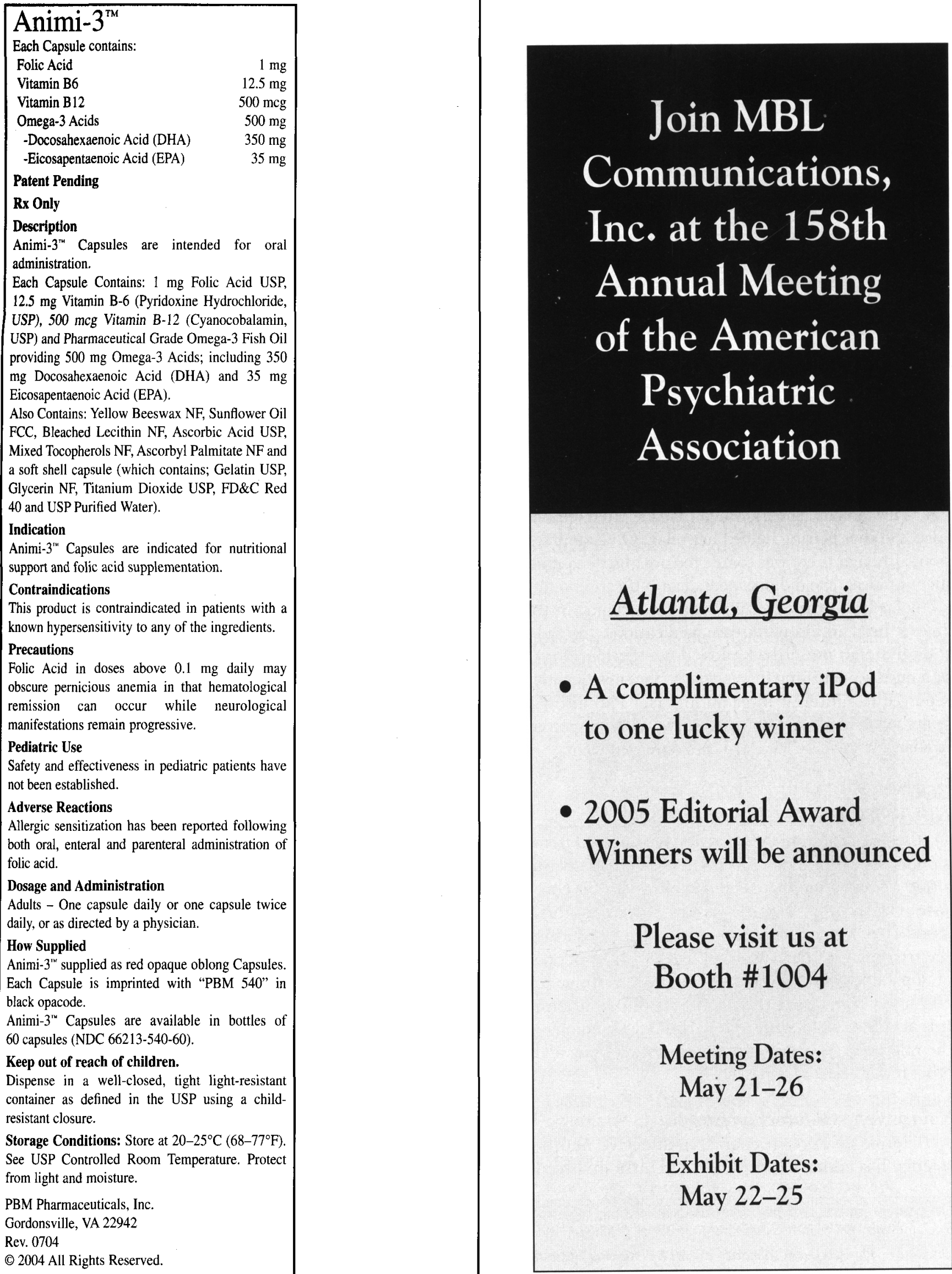

